\title{
OC-127 RISK FACTORS AND IMPACT OF CLOSTRIDIUM DIFFICILE INFECTION IN ACUTE SEVERE COLITIS NEEDING HOSPITALIZATION
}

doi:10.1136/gut.2011.239301.127

M Robinson," R Meigh, G Barlow, S Sebastian Hull \& East Yorkshire NHS Trust, Hull, UK

Introduction Limited data is available on the disease specific risk factors and the impact of Clostridium difficile when patients are admitted with a flare up of ulcerative colitis. We aimed to study a cohort of hospitalised patients admitted with acute severe colitis with concomitant $C$ difficile infection in order to identify the disease specific risk factors and clinical outcomes.

Methods From microbiology database over a 5 -year period containing all $C$ difficile positive results, patients with an established diagnosis of ulcerative colitis were included. Control group consisted of patients with acute severe colitis admitted to hospital during the same time period with a negative $C$ difficile toxin assay result during the index admission. Demographic features, presence of predetermined risk factors, duration of hospital stay and outcomes were reviewed.

Results 2612 patients with positive $C$ difficile toxin were identified over the 5 year period of which 48 (1.8\%) had preexisting established diagnosis of ulcerative colitis. Among patients with UC and C. difficile infection (group A), 3 (6.3\%) patients were tested to have positive $C$ difficile toxin in outpatients preadmission. The remaining 45 (one patient excluded) had a positive test during hospitalisation. When compared with controls of UC patients without C. difficile (group B), group A patients were of older age (mean 44.6 years vs. 29.3 years, $p=0.001$ ), had a longer history of colitis (mean 6.2 years vs 2.4 years, $p=0.01$ ) and more had preexisting pan colitis (36/47 vs $23 / 47, p=0.041)$. In addition, patients in group A had higher frequency of antibiotic use within six months ( $72 \%$ vs $15 \%, p=0.001)$ and a higher proportion had hospitalizations within the previous 6 months (26/47 vs $7 / 47, p=0.001)$. There was no significant difference in use of steroids, 5 ASAs, thiopurines and biologicals. The differentiating clinical features at admission in those with C. difficile were the presence of fever and abdominal pain. Pseudo membrane was recorded in 2 of 27 patients who had sigmoidoscopy. The duration of hospital stay was higher in group A (median 16 days, range 5-43 days) when compared to group B (median 8 days, range 4- 14 days). There was no difference in colectomy rates with two patients from group A and three patients from group B undergoing colectomy. There was no mortality in either group. 
Conclusion Clostridium difficile infection in admitted patients with ulcerative colitis leads to prolonged hospital stay but does not seem to increase mortality or colectomy rates. In addition to the known risk factors in general population, duration and extend of colitis may be additional risk factors for $C$ difficile infection in patients with UC. The relevance of testing for $C$ difficile during flare up of colitis in an outpatient setting is unclear.

Competing interests None. 